\title{
B IDENTIFICATION BY TOPOLOGY WITH THE SLD DETECTOR*
}

\author{
W. B. ATWoob \\ Stanfond Linear Accelerator Center. \\ Stanfond University, Stonfond, California 94909
}

\section{INTRODUCTION}

Al both the SIC and LEP (arge samples of $2^{0} \rightarrow$ bi will soon be available. The challenge is to find experimental techninues to inelusively tag and idemify the varions particles $b$ quarku may fragment into. The work described here makes use of the new geveration of close-in tracking devices (commonly called vertex detectors) which will acliewe unparalleled precision in particle trajectory tesolution. Sonse of these derices (notably in ALEPH at LEP and SLD at the 3L.C) provide threedimensional information which is crucial in solving the pattern recognition problen discussed below. In the case where only two-dimensional information is avnilable other than tracking infornsation (e.g., particle identification) may be required. The problem to solve is shown in Fig. $\mathrm{l}$ (a). The tracking system is used to extrapolate Iracks into some preselected point (e.g., distance of closeat approach to the bram line, a fix radius, elc.). The result is a picture of many crossing tracks and the problem is to find the real vertices among all the possible combinations. The Monte Carlo input which resulted in the "bundle-of-sticks" shown in Fig. 1(a) is shown in Fig. l(b).

Contributed to thr Workshop on B. Pactores and Relnied Issurs, Blois, Finnce, June 96-July 1, 10k9,

- Work supported hy Department of Hatergy contract DE-AC03-705F00515. 


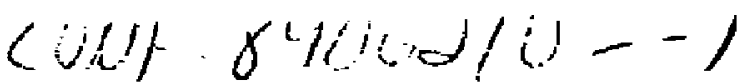

The algorithm 1 developed to study $B$ physics with the SLD Detector relies on the three-dimensional precision reconstruction of the tracks. This is the tool which provides for the resolution of anbiguities.

To start, pairs of tracks are subjected to an opening-angle verse total moment um cut. By requiring $\cos \theta_{\text {apen }}>0.2$ only tracks in the same jet may be paired." $\lambda$ total momentum requirement of at least $>0.5 \mathrm{GeV} / \mathrm{c}$ eliminates low momentun combinations which bave a poor signal-to-noise ratio. I also placed a correlatel rut at $\cos \theta_{\text {open }}$ vs. Frot of the form:

$$
\text { Pror }<\frac{(0.4 \mathrm{GeV} / \mathrm{c})}{1-\cos \theta_{\mathrm{open}}} \text { for } \cos \theta_{\mathrm{open}}<0.95 \text {. }
$$

This cut eliminntes paits of excessively high mass and may cause a slight iner. ficiency in the detection of some rare $B$ decays (e.g.. $B_{d} \rightarrow \pi^{+} \pi^{-}$) but the signal. to noise tatio in the region eliminated by this cut is extremely poor. A scallet filot of track pairs which come from the same vertex is shown in Fig. 2(a). The culs are indicated on this figure, as well. Wrong combinations are shown in Fjg. 2(b).

The "vertex" of the track pair is assigned to lie on the line of the distance of closest approarh (DOCA) between them, weighted by each track's momemtum so as to favor the higher momentum track. This weighing is used as most tracks are multiple scattering linited. The ${ }^{*}{ }^{2 n}$ for the association is formed:

$$
x_{v T X}^{2}=\sum_{\text {track. }}\left(\frac{d_{i}}{\sigma_{1}}\right)^{2}
$$

where

$$
d_{i}=\mid \bar{x}_{1}(\text { DOCA })-\bar{x}_{v T \times} \mid
$$

and $n_{1}$ is the estimated tracking precision, and tor the SLD is:

$$
0, \approx 10 \mu \mathrm{m} \oplus \frac{70 \mu \mathrm{m} \mathrm{GeV}}{P_{\text {trat } / \mathrm{GeV}}}
$$

A cut on $x_{v T x}^{2}$ is made: $x_{v r x}^{2}<9$. The separation of the vertex from line bram collision point is calculated next. In the case of the SLC. the location each beam

t One advantage of doing this phytito al the $Z^{0}$ over the $T_{\text {th }}$ is that fon the mont part the jorts conisin fragments from juct one or the other 6 quaks. 
crossing is known to about $30 \mu \mathrm{m}$ and the spot size is $4 \mu \mathrm{m}$. Thus there is megligilsle uncertainty in the origin of the flight paths. The flight palh is "signed" $+(-)$ arcording 10 whether the momentum of the vertex points away from (towards) the primary vertex point. As the dislance between the primary vertex and the secondary vericx decreases, the number of combinations increases rapidly. A cut is made to require the fight path to be greater than $500 \mu \mathrm{m}$. A good sigal-to-noise ratio is thus arbieved at the expense of about $30 \%$ in efficiency. This is the single largest inefficiency in this vertexing algorithm. The signal and background plots for this cut are shown in Figa. $3(a)$ and $3(b)$.

All combinations of track puirs salisfying the above criterion are temporarily retained. Hence a vertex with four charged prongs may result in up to aix track pairs. The track pairs are now used as "seeds" wo form vertices of higher prong count. The procedure used is to first find the next clowest track in the same jet [cos $\theta_{\text {open }}$ (vertextrack) $>0$ ). Closenes is menured in normalized units $\left(\sigma^{\circ}{ }^{\prime}\right)$ to svoid momentum dependencies. If a track ja within 3.5 a an attempt is made to incorporate it into the vertex. A new vertex lucation is computed such that

$$
x^{2}=\sum_{\text {tracke }} \frac{\left(\bar{x}_{i}-\bar{x}_{v j x}\right)^{2}}{\sigma_{i}^{2}}
$$

is minimized; $\bar{z}_{i}$ is the space point on the track at the dintance of closest approuch to the vertex point $\vec{x}_{v i x}$, and $\sigma_{i}$ is the projected revolution along the distance of closeat approach vettor. The new vertex is accepted if $x^{2}<6.3$ and the new flight path $\left|\vec{x}_{v r x}\right|$ is greater than $500 \mu \mathrm{m}$. If it pases these criterion and has not been previously cataloged it is temporarily stoned awoy. This process of adding tracks continues until no more tracks pas the procedure outlined above.

The temporary vertices are grouped by prong count and teated for net charge, (|Q aet $\mid \leq 1)$, strangenew $\left(\left|S_{\text {ant }}\right| \leq 1\right)$, and baryon count $\left(\left|B_{\text {oet }}\right| \leq 1\right)$. The last two criterion rely on the particle identification. Vertices passing these cuts have their apparent mass, four vector, impact paraneter with respect to the beam line, the dintance to the next close track in the event, and various quant um numbers tabulated and stored away in the output banks. 
Cascade topologies are a common occurzence in $B$ decay and may be found hy iterating the vertex find proress. The starting "track pairs" now contain a trark and a "pseudo track" defined by a vertex found in the first pass. The frst pasy vertices ane presumed to be the tertiary (charmed) vertex.

The only new criterion applied is to require that the new (secondary) vertex and the tertivey vertex be separated by at least $150 \mu \mathrm{m}$ and that the secondery vertex is closer to the primary verlex than the tertiary

In both passes of vertex finding no attempt is made to resolve ambiguities; tracks can be (and often are) asociated with more than one vertex.

The efficiency for finding cascade topologies from $B$ mesons is $\mathbf{b}-10 \%$ depending on the cuts used. This is to be compared with the $30 \%$ efficiency tor the first pass vertex finding on single vertices.

In another contribution to this conlerence, ${ }^{1}$ a detailed breakdown of the cascade topologies a to origin is given. Examination of the entries in Table 1 (Ref. 1) sorted by the charge of the vecondary and tertiary vertices show a furorable distinction between $B_{*}^{+}, B_{-}^{-}, B^{0}$ and $B$. Separation of $B_{t}$ and $B_{a}$ in the $B^{0}$ sample requires particle identification.

\section{Referencea}

1. Arwood, W. B. AND B. Mouns. B physics at the $Z^{0}$ pole. SLAC-PuB5048 (Auguat 1989). 
Table I Breakdown of found cascade topology events by secondary and tertiary vertex changes, $Q_{t}$ and $Q_{c}$. For each charge combination, the sample is resolved into particles and antiparticles, as well as the meson flavors $B_{m}, B_{2}$, and $B_{1}$ (see upper left entries under $Q_{b}=Q_{c}=-1$ for key) by referring baik to the Monte Carlo input.

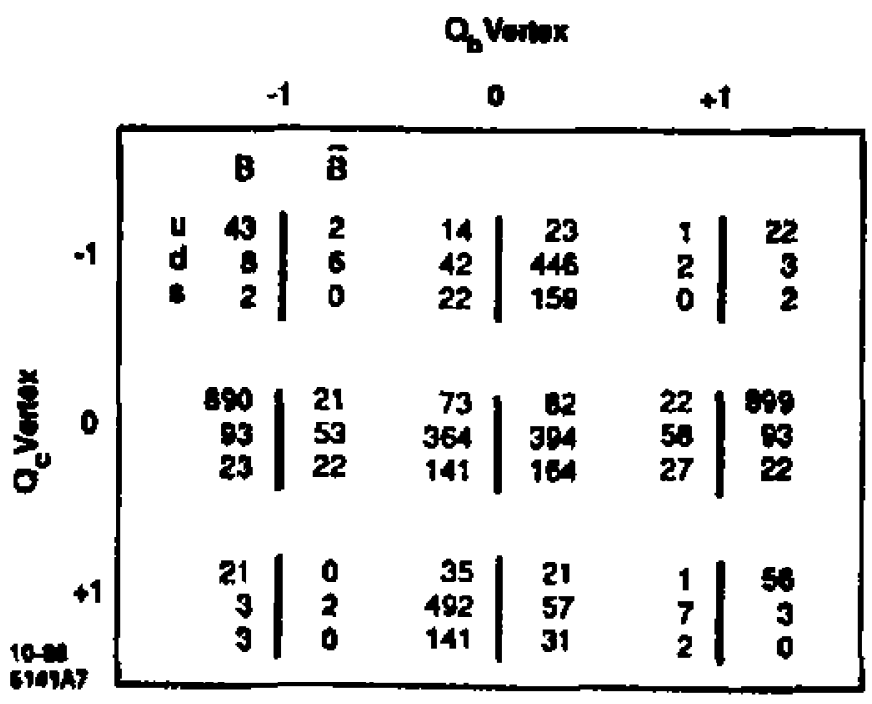




\section{Figure Captions}

Fig. 1. A Lund Monte Carlo event of $Z^{0} \rightarrow b 5$ : (a) the simulated reconbtructed tracks extrapolaled into a radius of $1 \mathrm{~mm}$ about the beam line; (b) the input Monte Carlo event is shown with the tracks properly asociated with their vertices.

Fig- 2. Scatter plots of $P_{\text {TOT }}$ vs. $\cos \theta_{\text {open }}$ for all track pairs: (a) only correctly associaled pairs are shown; (b) only incorrectly associated pairs are plotted.

Fig- 3. Plots of the signed flight palh from the primary vertex to the pair vertex: (a) only correctly taken pairs are shown; (b) only wrong combinations are shown. 

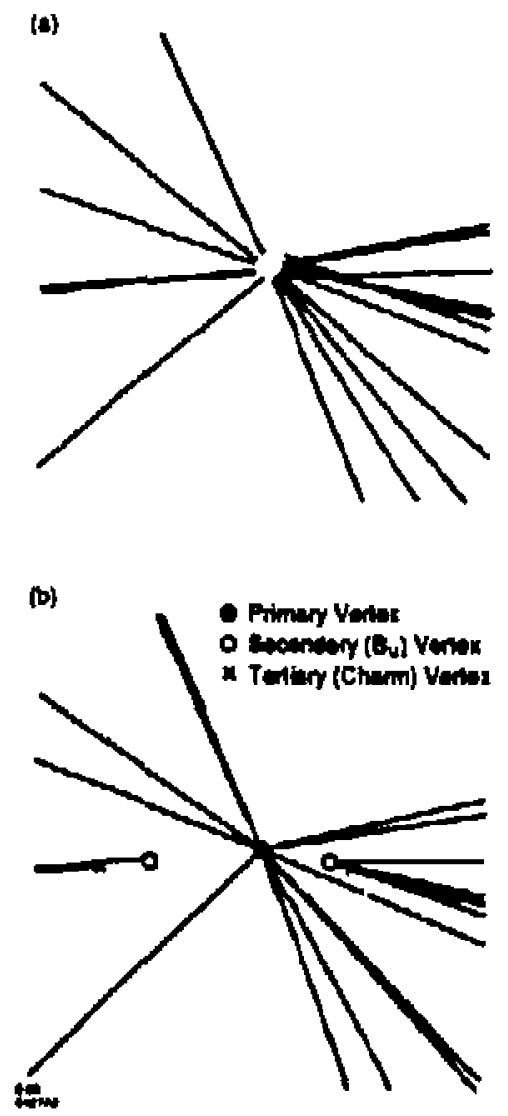

Fig 1 


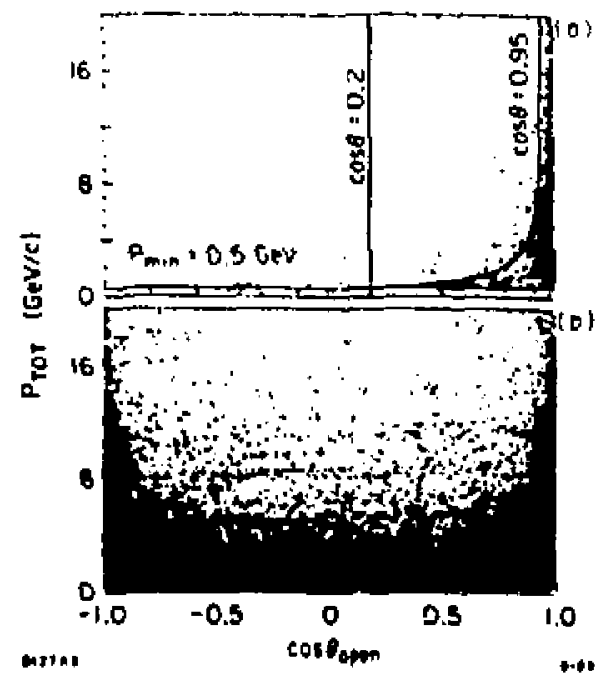

Fig. 2

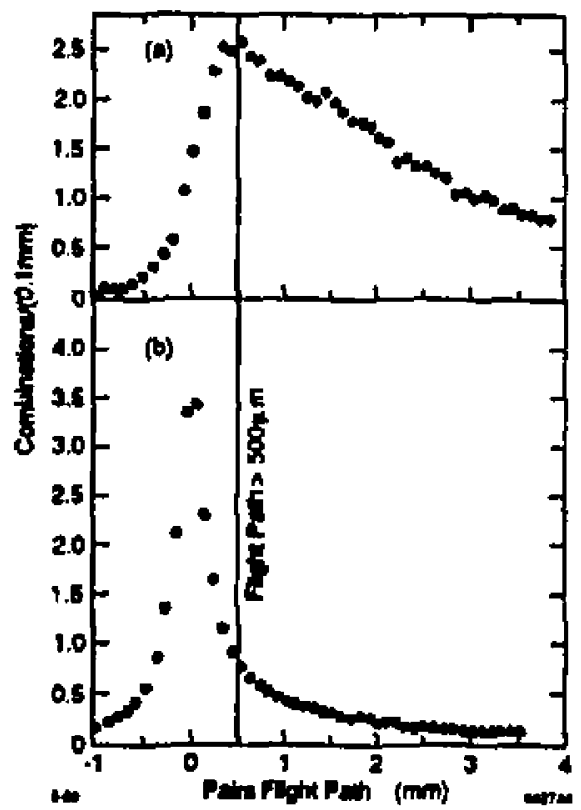

Fig. 3 